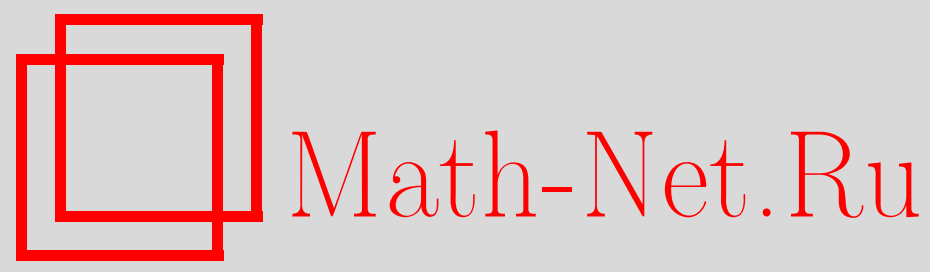

В. С. Монахов, Е. Е. Грибовская, О максимальных и силовских подгруппах конечных разрешимых групп, Матем. заметки, 2001, том 70, выпуск 4, 603-612

DOI: https://doi.org/10.4213/mzm772

Использование Общероссийского математического портала Math-Net.Ru подразумевает, что вы прочитали и согласны с пользовательским соглашением http://www.mathnet.ru/rus/agreement

Параметры загрузки:

IP : 44.207 .124 .84

26 апреля 2023 г., $17: 28: 40$

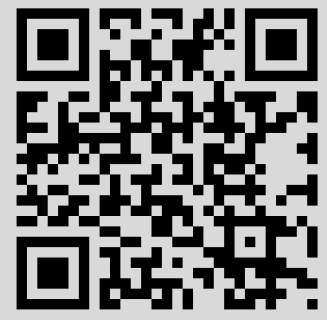




\title{
О МАКСИМАЛЬНЫХ И СИЛОВСКИХ ПОДГРУППАХ КОНЕЧНЫХ РАЗРЕШИМЫХ ГРУПП
}

\author{
В. С. Монахов, Е. Е. Грибовская
}

Исследуется строение конечных разрешимых групп, у которых каждая силовская подгруппа является произведением двух циклических подгрупп. В частности, доказывается, что нильпотентная длина таких групп не превосходит 4. Устанавливается также, что нильпотентная длина конечной разрешимой группы, в которой индекс каждой максимальной подгруппы есть либо простое число, либо квадрат простого числа, либо куб простого числа, не превосходит 5 .

Библиография: 11 названий.

Согласно теореме Цассенхауза [1, теорема IV .2.11] коммутант конечной группы с циклическими силовскими подгруппами является циклической холловой подгрупшой, факторгруппа по которой также циклическая. Поэтому нильпотентная длина таких групп $\leqslant 2$. Конечным группам с метациклической силовской 2 -подгруппой посвящена работа Мазурова [2]. Композиционные факторы неразрешимых груп с метациклическими силовскими $p$-подгруппами для всех $p$ перечислены в [3].

Биииклической назьвают конечную группу, являющуюся произведением двух циклических подгрупп. Метациклическая группа всегда является бициклической. Обратное утверждение верно для примарных групп нечетного порядка и не всегда вьполняется для бициклических 2-групп [1, теорема III.11.5].

В теоремах 1 и 2 настоящей заметки исследуется строение конечной разрешимой группы с бициклическими силовскими подгруппами. В частности, устанавливается, что нильпотентная длина таких групा $\leqslant 4$. Поскольку в любой конечной разрешимой группе индекс каждой максимальной подгрупшы есть степень простого числа, то предложенный подход к доказательству теорем 1, 2 оказался применимьм и к изучению конечных разрешимых групп, у которых индекс каждой максимальной подгруппы либо простое число, либо квадрат простого числа, либо куб простого числа. Строение таких групा рассматривается в теореме 3 , где, в частности, доказьвается, что нильпотентная длина этих групп $\leqslant 5$.

Рассматриваются только конечные групы. Напомним используемые определения и обозначения. Пусть $A_{G}$ - наибольшая нормальная подгрупа группы $G$, содержащаяся в подгруппе $A$, а $\Phi(G)$ - подгруппа $\Phi$ раттини групшы $G$. Запись $G=A B$ означает, что группа $G$ есть произведение своих подгруп $A$ и $B$, а $G=[A] B$ - полупрямое произведение с нормальной подгруппой $A$. Через $n(G), d(G)$ и $l_{p}(G)$ обозначаются нильпотентная длина, производная длина и $p$-длина групшы $G$ соответственно, a $r(X)$ - главньй ранг 
разрешимой группы $X$, см. [1, с. 712]. Информация о бишиклических группах содержится в [4]. Отметим, что существует бишиклическая группа порядка 16, которая не является метациклической. Кроме того, в отличие от метациклических групп, подгруппы бишиклических 2-групп не всегда бишиклические. Ясно, что факторгрупшы бициклических групп бициклические.

Для доказательства теорем потребуются следующие леммы.

Лемма 1. Пусть $P$ - бичиклическая р-группа и $N$-ее дополняемая нормальная noдzpynna. Tогда

(1) если $p=2$, mo $|N / \Phi(N)| \leqslant 4$;

(2) если $p>2$, то либо $N=P$, либо $N$ чиклическая.

ДоКАЗАТЕЛЬСТВо индукцией по порядку группы.

(1) Допустим противное, пусть $P=A B$ - бищиклическая 2-групша, являющаяся произведением двух своих циклических подгрупп $A$ и $B$, и пусть $N$ - нормальная подгруппа, имеющая дополнение $K$ в группе $P$. Воспользуемся методом доказательства леммы из [5]. Если подгруппа $\Phi$ раттини $\Phi(N)$ отлична от единичной подгрупшы, то по индукции $|N / \Phi(N)|=2$ или 4. Пусть $\Phi(N)=1$. Тогда $N$ - элементарная абелева группа, а так как $N$ нормальна в $P$, то пересечение $N \cap Z(P)$ с центром отлично от 1 . Для подгруппы $T$ порядка 2 из этого пересечения по индукции $|N / T| \leqslant 4$, поэтому можно считать, что $|N|=8$. Пусть $C=C_{P}(N)$. Тогда $C$ нормальна в $P$ и если $L=C \cap K \neq 1$, то по индукции подгрупа $N L / L \simeq N$ имеет порядок 2 или 4 . Поэтому следует считать, что $N=C$. Теперь $P / N$ изоморфна подгруппе группы автоморфизмов групшы $N$, которая изоморфна $G L(3,2)$, и $K$ изоморфна подгруппе группы диэдра порядка 8 ; в частности, экспонента $K$ делит 4. Если $n k^{-1}$ - произвольный элемент группы $P, n \in N, k \in K$, то $\left(n k^{-1}\right)^{4}=\left(n k^{-1} n k^{-1}\right)\left(n k^{-1}\right)^{2}=n n^{k} n^{k^{2}} n^{k^{3}}=n^{(1+k)^{3}}=1$, т.е. группа $P$ имеет экспоненту 4. Поэтому $|P|=16,|A|=|B|=4, A \cap B=1$. Теперь центр $Z=Z(P)$ - нециклическая подгрупша порядка 4 по [1, теорема VI.10.1]. Группа $G$ имеет разложение в смежные классы по центру

$$
G=Z \cup n Z \cup k Z \cup n k Z
$$

где $n \in N \backslash Z,\langle k\rangle=K$. В $Z, n Z, k Z$ все элементы имеют порядок 2, поэтому образуюшие элементы $a$ и $b$ подгрупп $A$ и $B$ содержатся в смежном классе $n k Z$. Но если $a=n k z_{1}, b=n k z_{2}$, то $a^{2}=(n k)^{2}=b^{2}$ и $A \cap B \neq 1$, противоречие.

(2) Пусть $p>2$ и $P=[N] K$ - метациклическая $p$-группа наименьшего порядка, разложимая в полупрямое произведение нормальной подгруппы $N$ и подгруппы $K$, и пусть $P \neq N$. По индукции $\Phi(N)=1$, поэтому $N$ - элементарная абелева подгруппа порядка $p^{2}$. Пусть $b$ - элемент простого порядка из $K$. Если $P_{1}=[N]\langle b\rangle-$ собственная подгруппа в $P$, то по индукции в группе $P_{1}$ подгруппа $N$ недополняема, противоречие. Значит, $K=\langle b\rangle$ и $P$ - группа порядка $p^{3}$. Ясно, что $P$ неабелева. Если $P$ содержит нормальную подгруппу $D$ порядка $p$ с циклической факторгруппой $P / D$, то $D$ содержится в центре $P$ и $P$ абелева, противоречие. Следовательно, $P$ содержит циклическую подгруппу индекса $p$ и подгруппа $\Omega_{1}(P)$, порожденная элементами порядка $p$, является элементарной абелевой подгруппой порядка $p^{2}$ по [6, теоремы 5.4 .3 и 5.4.4]. Теперь $N=\Omega_{1}(P)$ и подгруппы $K$ порядка $p$ не сушествует. Значит, допушение неверно и лемма справедлива. 
Лемма 2. Пусть $G$ - p-разрешимая группа с бициклической силовской p-подгруппой. Тогда

(1) если $p=2$, то $G / O_{2^{\prime}, 2}(G)$ либо имеет нечетный порядок, либо изоморфна $S_{3} ;$ в частности, $l_{2}(G) \leqslant 2$;

(2) если $p>2$, mo $l_{p}(G) \leqslant 1$.

ДокАЗАТЕЛЬСТво. (1) По индукции можно считать, что подгруппа Фиттинга $F$ является 2-группой. Пусть $\Phi$ - подгруппа $\Phi$ раттини групшы $G$. Так как по [1, теорема III.4.5] факторгруппа $F^{*}=F / \Phi$ является подгруппой Фиттинга группы $G^{*}=G / \Phi$ и $F^{*}$ - прямое произведение минимальных нормальных подгрупп группы $G^{*}$, то $F^{*}=$ $C_{G^{*}}\left(F^{*}\right)$ и $F^{*}$ дополняема в группе $G^{*}$ по [1, лемма III.4.4]. По лемме 1 порядок подгруппы $F^{*}$ равен 2 или 4. Если $\left|F^{*}\right|=2$, то $F^{*}=G^{*}$ и $G-2$-группа. Если $\left|F^{*}\right|=4$, то $G / F \simeq G^{*} / F^{*}$ изоморфна подгруппе группы $G L(2,2) \simeq S_{3}$ и либо $|G / F|$ нечетен, либо $G / F \simeq S_{3}$

(2) По индукции подгруппа Фраттини единична и в групше нет неединичных нормальных $p^{\prime}$-подгруп. Кроме того, в группе единственная минимальная нормальная подгруппа. Поэтому $P=O_{p}(G)$ - элементарная абелева $p$-подгруппа, совпадающая со своим централизатором и имеюшая в $G$ дополнение. Поскольку силовская $p$-подгрупша в группе $G$ метациклическая, то порядок $P$ равен $p$ или $p^{2}$. Если $|P|=p$, то $G / P$ - циклическая группа порядка, делящего $p-1$, и $P$ силовская в $G$. Если $|P|=p^{2}$, то по лемме 1 подгруппа $P$ опять силовская в $G$. Лемма доказана.

Сверхразрешимым корадикалом групы называется ее наименьшая нормальная подгруппа, факторгруппа по которой сверхразрешима. Легко проверить, что сверхразрешимый корадикал в любой группе определен однозначно, а если $H$ и $K$ - подгруппы группы $G$ и $H \leqslant K$, то сверхразрешимый корадикал подгрупшы $H$ содержится в сверхразрешимом корадикале подгрупшы $K$, см. [7, с. 10].

ЛЕмма 3. Пусть $H$ - неприводимая разрешимая подгруппа группы $G L(2, p)$. Tогда

(1) $r(H) \leqslant 2, n(H) \leqslant 3 u d(H) \leqslant 4$;

(2) сверхразрешимый корадикал подгруппы $H$ является расширением чиклической 2-группы порядка, делящего $(p-1)$, с помощью подгруппы из әлементарной абелевой группы порядка 4;

(3) если $H-3^{\prime}$-группа, то $H$ сверхразрешима.

ДокАЗАТЕЛЬСТво. Заключим $H$ в максимальную разрешимую подгруппу $M$ группы $G L(2, p)$ и докажем утверждение леммы для $M$. Ясно, что тогда утверждение будет справедливо и для $H$. Очевидно, $M$ - неприводимая подгруппа.

Если $M$ импримитивна, то $M$ изоморфна сплетению циклической групшы $A$ порядка $p-1$ и группы $B$ порядка 2, см. [8, теорема 18.5]. Диагональ $S$ базы $D=A \times A$ этого сплетения будет нормальной подгруппой в $M$, изоморфной $A$, поэтому $M$ обладает нормальным рядом $1 \triangleleft S \triangleleft D \triangleleft M$, где групшы $S$ и $D / S$ циклические порядка $p-1$ и $M / D$ порядка 2 . Поэтому $M$ сверхразрешима и $d(M) \leqslant 2$.

Если $M$ примитивна и ее максимальная абелева нормальная подгруппа $F$ изоморфна мультипликативной групше поля $G F\left(p^{2}\right)$, то по [8, теорема 21.1] факторгруппа $M / F$ имеет порядок 2 и опять $M$ сверхразрешима. Ясно, что $d(G) \leqslant 2$. 
Пусть $M$ примитивна и ее максимальная абелева нормальная подгруппа $F=$ $G F(p)^{\#} E_{2}$. Подгруппа $F$ состоит из скалярных матриц [8, с. 234, формула (13)], поэтому $F$ содержится в центре $M$. В $M$ существует нормальная подгруппа $A$ такая, что $A$ содержит $F$ и $A / F$ изоморфна элементарной абелевой групше порядка 4 , а $M / A$ изоморфна симметрической группе степени 3 , см. [8, с. 234, формулы (13) и (14)]. Заметим, что подгруппа $M$ нильпотентна, если ее порядок не делится на 3 . Так как $r(M / A)=1$, $r(A / F)=2$ и $F$ нормальна в $M$, то $r(M / F) \leqslant 2$. Из того, что $F$ содержится в центре $M$ и $r(F)=1$, следует, что $r(M) \leqslant 2$.

Так как $A$ нильпотентна, то $n(M) \leqslant 3, d(M) \leqslant 4$ и силовская 2 -подгруппа $T$ из $A$ нормальна в $M$. Теперь факторгруппа $M / T$ обладает нормальным рядом $1 \triangleleft A / T \triangleleft M / T$, который уплотняется до главного ряда с факторами простых порядков. Значит, $M / T$ сверхразрешима и сверхразрешимьй корадикал подгруппы $M$ содержится в $T$. Но $A=T F$, поэтому $A / F \simeq T / T \cap F$ и $T$ является расширением циклической групш $T \cap F$ порядка, делящего $p-1$, с помошью элементарной абелевой групшы $A / F$ порядка 4 . По $[8$, гл. V, $§ 21]$ других ситуаций для $M$ нет. Лемма доказана.

Лемма 3 обобщает лемму [9, лемма 1$]$.

ЛЕмма 4. Пусть $H$ - неприводимая разрешимая подгруппа группы $G L(3, p)$. Тогда

(1) $r(H) \leqslant 3, n(H) \leqslant 4 u d(H) \leqslant 5$;

(2) для сверхразрешимого корадикала $U$ подгруппы $H$ возмохнны два случая:

(2.1) подгруппа $U$ абелева порядка, делящего $(p-1)^{2}$;

(2.2) U-3-замкнутая $\{2,3\}$-подгруппа; ее силовская 3-подгруппа является расширением ииклической группы порядка, делящего $p-1$, с помощью подгруппы из әлементарной абелевой группь порядка 9, а силовская 2-подгруппа является расширением ииклической группы порядка, делящего $p-1$, с помощью подгруппь из әлементарной абелевой группь порядка 4.

ДокАЗАТЕЛЬСтво. Заключим $H$ в максимальную разрешимую подгруппу $M$ группы $G L(2, p)$ и докажем утверждение леммы для $M$. Ясно, что тогда утверждение будет справедливо и для $H$. Очевидно, $M$ - неприводимая подгруппа. По теореме Супруненко [8, с. 234] возможны три случая: группа $M$ импримитивна; групша $M$ примитивна и ее максимальная нормальная подгруппа $F$ изоморфна мультипликативной группе поля $G F\left(p^{3}\right)$; группа $M$ примитивна и ее максимальная нормальная подгруппа $F=G F(p)^{\#} E_{3}$.

В первом случае группа $M$ мономиальна и согласно [8, теорема 18.5] сопряжена в $G L(3, p)$ со сплетением $G F(p){ }^{\#} S_{3}$, где $S_{3}$ - симметрическая группа порядка 6, см. [8, c. 234]. Кроме того, группа $M$ состоит из линейных преобразований пространства $V=$ $V(3, G F(p))$ таких, что $g\left(v_{i}\right)=\lambda_{i} v_{i_{1}}$, где $\left\{v_{1}, v_{2}, v_{3}\right\}$ - базис $V$. По [1, теорема II.7.2] преобразования $d$, для которых $d\left(v_{i}\right)=\lambda_{i} v_{i}$, образуют абелеву подгруппу $D$ порядка $(p-1)^{3}$, нормальную в $M$, и $M / D \simeq S_{3}$. Поэтому $d(M) \leqslant 3$ и $r(M) \leqslant 3$. Теперь $K=D \cap S L(3, p) \triangleleft M$ и $|K|=(p-1)^{2}$. Ядро гомоморфизма $f: d \mapsto \operatorname{det} d$ совпадает с $K$. Поэтому $D / K$ - циклическая группа порядка $p-1$. Отсюда следует, что $M / K$ сверхразрешима и сверхразрешимый корадикал групшы $M$ является абелевой подгруппой порядка, делящего $(p-1)^{2}$. 
Пусть теперь $M$ примитивна и ее максимальная абелева нормальная подгрупа $F$ изоморфна мультипликативной групе поля $G F\left(p^{3}\right)$. Группа $F$ циклическая и $|F|=$ $p^{3}-1$. По [8, теорема 21.1] $|M|=3\left(p^{3}-1\right)$. Поэтому $M$ сверхразрешима и $d(M) \leqslant 2$.

Пусть $M$ примитивна и ее максимальная абелева нормальная подгруппа $F=$ $G F(p)^{\#} E_{3}$. Подгруппа $F$ состоит из скалярных матриц, поэтому $F$ содержится в центpe $M$. В $M$ существует нормальная подгруппа $A$ такая, что $A$ содержит $F$ и $A / F$ изоморфна элементарной абелевой группе порядка 9 , а $M / A$ изоморфна симметрической группе $S_{4}$ степени 4 , см. [8, с. 234 , формулы (13) и (14)]. Так как $A$ нильпотентна и $n\left(S_{4}\right)=d\left(S_{4}\right)=3$, то $n(M) \leqslant 4, d(M) \leqslant 5$. Поскольку $r\left(S_{4}\right) \leqslant 2$, то и $r(M) \leqslant 2$. Из того, что $M / A \simeq S_{4}$, следует, что $M$ содержит нормальную подгруппу $B$ такую, что $M / B \simeq S_{3}$ и $A \subseteq B, B / A \simeq E_{4}$ - элементарная абелева группа порядка 4.

Так как $A$ нильпотентна и нормальна в $M$, то $\{2,3\}$-холлова подгруппа $T$ из $A$ нормальна в $M$. Силовская 2-подгруппа в $T$ циклическая порядка, делящего $p-1$, а силовская 3-подгруппа $P$ в $T$ является расширением циклической подгруппы порядка, деляшего $p-1$, с помошю подгрупшы из элементарной абелевой группы порядка 9. Ясно, что $P$ нормальна в $M$.

Пусть $S-\{2,3\}$-холлова подгруппа в $B$. Так как $B=S F$ и $F \leqslant Z(M)$, то $S \triangleleft B$, поэтому $S \triangleleft M$. Пусть $Q$ - силовская 2 -подгруппа в $S$. Тогда $Q$ является расширением циклической подгрупшы порядка, деляшего $p-1$, с помошью подгрупшы из элементарной абелевой группы порядка 4. Так как $P \triangleleft M$, то $S=[P] Q$. Теперь ряд $1 \leqslant F S / S=A S / S=B / S \triangleleft M / S$ уплотняется до главного ряда с факторами простых порядков. Поэтому $M / S$ сверхразрешима и сверхразрешимьй корадикал $U$ подгруппы $M$ содержится в $S$. Таким образом, сверхразрешимьй корадикал $U$ является 3 -замкнутой $\{2,3\}$-подгруппй, силовская 3-подгруппа которой является расширением циклической группы порядка, делящего $p-1$, с помошью подгруппы из элементарной абелевой групшы порядка 9 , а силовская 2-подгруппа является расширением циклической группы порядка, деляшего $p-1$, с помощью подгрупшы из элементарной абелевой группы порядка 4. Лемма доказана.

Метанильпотентной назьвают группу, содержащую нильпотентную нормальную подгрупшу, факторгруппа по которой нильпотентна.

ЛЕмма 5. Если $G$-метанильпотентная группа, то $l_{p}(G) \leqslant 1$ для любого просmoro $p$.

ДоКАЗАТЕЛЬСТво. Пусть $N$ - нильпотентная нормальная подгруппа групшы $G, ф а к-$ торгруппа по которой нильпотентна. Для любого простого $p$ группа $N$ представима в виде $N=N_{p} \times N_{p^{\prime}}$, где $N_{p}$ - силовская $p$-подгруппа группы $N$, а $N_{p^{\prime}}$ - ее дополнение. Так как $N_{p^{\prime}} \triangleleft G$, то $G_{p} N_{p^{\prime}} \triangleleft G$, где $G_{p}$ - силовская $p$-подгруппа групшы $G$, поэтому $l_{p}(G) \leqslant 1$.

Теорема 1. Если $G$ - разрешимая группа с бициклическими силовскими подгруппами, то сверхразрешимый корадикал группы $G$ является расширением ниль-

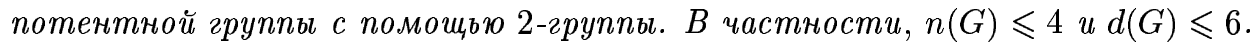

ДокАЗАТЕльство. Обозначим через $K$ сверхразрешимый корадикал групшы $G$ и применим индукцию по порядку группы $G$. Можно считать, что $\Phi(G)=1$ и в группе $G$ единственная минимальная нормальная подгруппа. Кроме того, подгруппа Фиттинга $F$ групшы является минимальной нормальной подгруппой, обладающей дополнением в групше $G$. Подгруппа $F$ совпадает со своим централизатором в группе $G$ и $G / F$ 
изоморфна подгрупше из группы автоморфизмов группы $F$. По лемме 1 всегда $|F|$ делит квадрат некоторого простого числа.

Если порядок $F$ - простое число $p$, то $G / F$ циклическая и $K$ содержится в $F$, поэтому подгрупша $K$ нильпотентна.

Если $|F|=p^{2}$, то $G / F$ изоморфна подгруппе групшы $G L(2, p)$ и по лемме 3 подгруппа $K F / F$ является 2 -группой. Так как $K F / F \simeq K / K \cap F$, то $K$ является расширением нильпотентной группы $K \cap F$ с помошью 2-группы $K F / F$. Отсюда, в частности, вытекает, что $n(G) \leqslant 4$.

Для оценки производной длины опять применим индукцию по порядку группы. По индукции можно считать, что в групше $G$ единственная минимальная нормальная подгруппа и подгруппа Фиттинга $F$ является $p$-группой для некоторого простого $p$. Заметим, что $d(F) \leqslant 2$ по [1, теорема VI.4.4]. Пусть $\Phi-$ подгруппа Фраттини группы $G$. Тогда $F / \Phi=F^{*}$ - подгрупша Фиттинга группы $G / \Phi=G^{*}$ и порядок $F^{*}$ равен $p, p^{2}$ или 8. Для нечетного $p$ это следует из того, что $F$ метациклическая, а для $p=2$ из того, что нормальные подгрупшы бициклических 2-групш порождаются не более чем тремя элементами [4]. Так как $C_{G^{*}}\left(F^{*}\right)=F^{*}$, то $G^{*} / F^{*}$ изоморфна $G / F$ и изоморфна подгруппе групшы автоморфизмов $F^{*}$. Если $\left|F^{*}\right|=p$, то $G / F$ циклическая и $d(G) \leqslant 3$. Если $\left|F^{*}\right|=4$, то $|G / F|$ делит 6 и $d(G) \leqslant 4$. Если $\left|F^{*}\right|=p^{2}$ и $p>2$, то $d(G) \leqslant 6$ по лемме 3 . Если $\left|F^{*}\right|=8$, то $G / F$ изоморфна разрешимой подгруппе группы $G L(3,2)$ и $d(G) \leqslant 5$. Таким образом, всег да $d(G) \leqslant 6$. Теорема 1 доказана.

Если $\pi$ - некоторое множество простых чисел, то $\pi^{\prime}-$ множество всех простых чисел, не принадлежащих $\pi$. Через $G_{\pi}$ обозначается $\pi$-холлова подгруппа групшы $G$.

Лемма 6. В разрешимой группе с биииклическими силовскими 2- и 3-подгруппами $\{2,3\}^{\prime}$-холлова подгруппа нормальна.

ДокАЗАТЕЛЬСТво индукцией по порядку групшы. Пусть $G$ - разрешимая группа с бициклическими силовскими 2- и 3 -подгруппами и $\pi=\pi(G) \backslash\{2,3\}$. По индукции $O_{\pi}(G)=1$, подгруппа Фраттини единична и в группе $G$ единственная минимальная нормальная подгрупша $F$, которая является подгруппой Фиттинга. Кроме того, $F=C_{G}(F)$, факторгруппа $G / F$ изоморфна подгруппе группы автоморфизмов $F$ и $G=[F] M$ для некоторой максимальной в $G$ подгрупшы $M$. Если $F-2$-группа, то по лемме 1 порядок $F$ равен 2 или 4 и $G_{\pi}=1$. Если $F-3$-группа, то по лемме 2 подгруппа $F$ силовская в $G,|G / F|$ делит $|G L(2,3)|=2^{4} 3$ и опять $G_{\pi}=1$. Лемма доказана.

Аналогичное утверждение, но при условии, что силовские 2- и 3-подгрупшы метациклические, доказано в [3].

ТЕОРема 2. Пусть G-разрешимая группа с бичиклическими силовскими подгруппами. Тогда

(1) $\{2,3\}^{\prime}$-холлова подгруппа нормальна в группе;

(2) $2^{\prime}$-холлова подгруппа $G_{2^{\prime}}$ дисперсивна по Оре, имеет нильпотентный коммутант и $d\left(G_{2^{\prime}}\right) \leqslant 3$

(3) $3^{\prime}$-холлова подгруппа $G_{3^{\prime}}$ дисперсивна по Оре, имеет нильпотентный сверхразрешимый корадикал $и d\left(G_{2^{\prime}}\right) \leqslant 4$;

(4) $n\left(G_{\{2,3\}}\right) \leqslant 3 u d\left(G_{\{2,3\}}\right) \leqslant 4$;

(5) $\{2, p\}$-холлова подгруппа $G_{\{2, p\}}$ дисперсивна по Оре $и d\left(G_{\{2, p\}}\right) \leqslant 4$ для любого $p>3$. 
ДокАЗАТЕЛЬСтво. Утверждение (1) следует из леммы 6.

(2) Можно считать, что 2 не делит порядок групшы $G$. По индукции $\Phi(G)=1$ и $F=F(G)$ - единственная минимальная нормальная подгруппа групшы $G$, обладающая дополнением в $G$, и $F=C_{G}(F)$. По лемме 2 подгруппа $F$ - силовская $p$-подгруппа группы $G$ и $|F|=p^{2}$ или $p$. Если $|F|=p$, то $G / F$ - циклическая группа порядка, делящего $p-1$. Пусть $|F|=p^{2}$. Тогда факторгруппа $G / F$ изоморфна подгрупше полной линейной группы $G L(2, p)$, порядок которой равен $\left(p^{2}-p\right)\left(p^{2}-1\right)$. Поэтому $p$ - наибольший простой делитель порядка групш $G$. Так как $G / F$ - неприводимая $p^{\prime}$-подгруппа нечетного порядка группы $G L(2, p)$, то опять $G / F$ циклическая. Таким образом, в любом случае факторгруппа $G / F$ циклическая. Поэтому группа $G$ дисперсивна по Оре и имеет нильпотентньй коммутант. Так как в $G$ все силовские подгруппы метациклические, то коммутант $G^{\prime}$ метациклический и $d(G) \leqslant 3$.

(3) Можно считать, что 3 не делит порядок группы $G$. Так как $G_{\{2,3\}^{\prime}}$ - нормальная подгруппа групшы $G$, то $G$ дисперсивна по Оре. По индукции с использованием леммы 3 получаем, что сверхразрешимый корадикал групшы $G$ нильпотентен. Для оценки производной длины применим индукцию по $|G|$. По индукции в группе $G$ единственная минимальная нормальная подгруппа $N$. Поэтому $N-p$-подгруппа для наибольшего $p$, делящего порядок групшы $G$. По лемме 2 подгруппа $N$ силовская в групе $G$ и $N / \Phi(N)$ элементарная абелева порядка $p^{2}$. Теперь $G / N$ изоморфна неприводимой разрешимой $3^{\prime}$-подгрупше групшы $G L(2, p)$. Из доказательства леммы 3 следует, что либо $d(G / N) \leqslant 2$, либо $G / N$ нильпотентна. Но в группе $G$ все силовские подгруппы имеют производную длину не вьше 2 по [1, теорема VI.4.4], поэтому в любом случае $d(G / N) \leqslant 2$ и $d(G) \leqslant 4$.

(4) Обозначим $\{2,3\}$-холлову подгруппу групшы $G$ через $K$. Из леммы 2 следует, что $n(K) \leqslant 3$. Для оценки производной длины опять применим индукцию по порядку группы. Заметим, что по [1, теорема VI.4.4] производные длины силовских 2-подгрупп и 3 -подгруп не вьше 2 . По индукции в группе $K$ единственная минимальная нормальная подгруппа $N$. Если $N-2$-группа, то подгруппа Фиттинга $F-2$-группа. По [4] подгруппа $F$ порождается не более чем тремя элементами, а по теореме Бернсайда о базисе порядок силовской 3 -подгрупш группы $K$ будет равен 3 . Теперь в факторгрупе $K^{*}=K / F$ подгрупша Фиттинга $F^{*}$ имеет порядок 3 и $K^{*} / F^{*}-$ группа порядка $\leqslant 2$, т.е. $d(K / F) \leqslant 2$. Так как $d(F) \leqslant 2$, то $d(K) \leqslant 4$. Если $N-3$-групша, то подгруппа Фиттинга $F$ совпадает с силовской 3 -подгрупой по лемме 2 и $K / F$ изоморфна силовской 2 -подгруппе. Поэтому опять $d(K) \leqslant 4$.

(5) Пусть $p>3$. Из (1) следует, что силовская $p$-подгруппа группы $G_{\{2, p\}}$ нормальна в $G_{\{2, p\}}$, поэтому $G_{\{2, p\}}-p$-замкнута и $d\left(G_{\{2, p\}}\right) \leqslant 4$. Теорема 2 доказана.

СледСТВИЕ. Группа $G$ нечетного порядка с метациклическими силовскими подгруппами дисперсивна по Оре и имеет нильпотентный коммутант. В частности, $d(G) \leqslant 3$.

Согласно теореме Холла [1, теорема VI.9.4] группа разрешима, если индекс каждой ее максимальной подгруппы - либо простое число, либо квадрат простого числа. Каморников [9] установил, что сверхразрешимый корадикал такой грушшы $G$ является расширением нильпотентной групшы с помощью 2 -группы и главньй ранг $r(G / \Phi(G)) \leqslant 2$. Если среди индексов максимальных подгрупп имеется куб простого числа, то группа 
может быть неразрешимой. Из теоремы Гуральника [10], доказательство которой использует классификацию конечных простых групп, вытекает следующее утверждение: если $G$ - неразрешимая группа, у которой индекс каждой максимальной подгрупшы либо простое число, либо квадрат простого числа, либо куб простого числа, то $G / S(G) \simeq$ $P S L(2,7)$. Здесь $S(G)$ - наибольшая разрешимая нормальная подгруппа групшы $G$.

Теорема 3. Если $G$ - разрешимая группа, у которой индекс каждой максимальной подгруппы - либо простое число, либо квадрат простого числа, либо куб простого числа, то $r(G / \Phi(G)) \leqslant 3$ и сверхразрешимый корадикал группы $G$ является расширением метанильпотентной группы с помощью 2-группь.

ДокАЗАТЕЛьСтво. Вначале покажем, что $r(G / \Phi(G)) \leqslant 3$. Пусть $M$ - максимальная подгрупша групшы $G$ и $M_{G}=\bigcap_{x \in G} M^{x}$ - ее ядро. Тогда $G / M_{G}$ примитивна и в $G / M_{G}$ сушествует единственная минимальная нормальная подгруппа $N / M_{G}$ такая, что

$$
C_{G / M_{G}}\left(N / M_{G}\right)=N / M_{G} \text { и }\left[N / M_{G}\right]\left(M / M_{G}\right)=G / M_{G} .
$$

Отсюда следует, что $\left(G / M_{G}\right) /\left(N / M_{G}\right) \simeq M / M_{G}$ и изоморфна подгрупше из группы автоморфизмов грушшы $N / M_{G}$.

Так как

$$
|G: M|=\left|N / M_{G}\right|=\left|G / M_{G}: M / M_{G}\right|=\left|N / M_{G}\right|
$$

и $|G: M|$ - либо простое число, либо квадрат простого числа, либо куб простого числа, то $\left|N / M_{G}\right|$ - либо простое число, либо квадрат простого числа, либо куб простого числа.

Пусть сначала $\left|N / M_{G}\right|=p$, где $p$ - некоторое простое число. Тогда $M / M_{G}$ - циклическая группа порядка, делящего $(p-1)$. Очевидно, $G / M_{G}-$ сверхразрешимая группа и $r\left(G / M_{G}\right)=1$.

Пусть теперь $\left|N / M_{G}\right|=p^{2}$. Тогда $M / M_{G}$ изоморфна некоторой разрешимой неприводимой подгруппе группы $G L(2, p)$ и $r\left(M / M_{G}\right) \leqslant 2$ по лемме 3 . Так как $\left|N / M_{G}\right|=p^{2}$, то $r\left(G / M_{G}\right) \leqslant 2$.

Предположим, что $\left|N / M_{G}\right|=p^{3}$. Теперь $M / M_{G}$ изоморфна некоторой неприводимой разрешимой подгруппе групшы $G L(3, p)$ и $r\left(M / M_{G}\right) \leqslant 3$ по лемме 4 . Так как $\left|N / M_{G}\right|=p^{3}$, то $r\left(G / M_{G}\right) \leqslant 3$.

Итак, в любом случае $r\left(G / M_{G}\right) \leqslant 3$. Но $\Phi(G)$ есть пересечение ядер максимальных подгрупп групшы $G$, поэтому по лемме Ремака (см. [7, с. 13]) $r(G / \Phi(G)) \leqslant 3$.

Теперь, используя индукцию по порядку групы $G$, покажем, что сверхразрешимый корадикал $K$ групшы $G$ является расширением метанильпотентной групшы с помошью 2 -группы. Можно считать, что подгруппа Фраттини группы $G$ единична, а подгруппа Фиттинга $F=F(G)$ является единственной минимальной нормальной подгруппой группы $G$. Кроме того, $F=C_{G}(F)$ и $G=[F] M$, где $M$ - некоторая максимальная подгруппа групшы $G$. Отсюда следует, что $|F|$ равен $p$, либо $p^{2}$, либо $p^{3}$, где $p$ - простое число.

Пусть сначала $|F|=p$. Тогда $G / F$ - циклическая групша как группа автоморфизмов группы простого порядка $p$ и группа $G$ сверхразрешима.

Пусть теперь $|F|=p^{2}$. Тогда $G / F$ изоморфна некоторой неприводимой разрешимой подгрупше групшы $G L(2, p)$. По лемме 3 подгруппа $K F / F$ является 2 -групшой, а из того, что $F$ нильпотентна, следует, что подгрупша $K$ является расширением нильпотентной группы с помощью 2-групшы. 
Осталось рассмотреть случай, когда $|F|=p^{3}$. Тогда $G / F$ изоморфна некоторой неприводимой разрешимой подгруппе группы $G L(3, p)$ и по лемме 4 групша $K F / F$ либо нильпотентна, либо является 3 -замкнутой $\{2,3\}$-подгруппой и подгруппа $K$ является расширением метанильпотентной группы с помошью 2-группы. Теорема 3 доказана.

СлЕдСТВИЕ 1. Если $G$ - разрешимая группа, у которой индекс каждой максимальной подгруппь - либо простое число, либо квадрат простого числа, либо куб простого числа, то $n(G) \leqslant 5$.

СлЕДСТВИЕ 2. Если $G$ - разрешимая группа, у которой индекс кажсой максимальной подгруппь - либо простое число, либо квадрат простого числа, либо куб простого числа, то $l_{p}(G) \leqslant 2$ для любого простого $p$.

ДокАЗАТЕЛЬСТво. Из теоремы 3 следует, что группа $G$ обладает нормальным рядом $1 \triangleleft H \triangleleft K \triangleleft G$ с метанильпотентными факторами $H$ и $G / K$, а $K / H-2$-группа. Теперь с помошью леммы 5 получаем, что $l_{2}(G) \leqslant 3$, а $l_{p}(G) \leqslant 2$ для любого простого $p>2$.

Для получения оценки $l_{2}(G) \leqslant 2$ воспользуемся индукцией по порядку группы $G$. Можно считать, что $\Phi(G)=O_{2^{\prime}}(G)=1$, а подгруппа Фиттинга $F=F(G)$ групшы $G-$ единственная минимальная нормальная подгруппа группы $G$, которая является элементарной абелевой 2-группой, и существует дополнение $M$ к подгрупе $F$ в групе $G$, см. [1, лемма VI.6.9]. Так как $|F|=|G: M|$, то порядок $F$ равен 2,4 или 8. Если $|F|=2$, то $G=F$. Если $|F|=4$, то факторгрупша $G / F$ изоморфна подгруппе полной линейной группы $G L(2,2) \simeq S_{3}$ и $l_{2}(G) \leqslant 2$. Если $|F|=8$, то факторгруппа $G / F$ изоморфна разрешимой подгруппе полной линейной групшы $G L(3,2)$ и в $G / F$ нет нормальных неединичных 2 -подгрупп. Так как группа $G L(3,2) \simeq P S L(2,7)$, то по [1, теорема II.8.27] либо $|G / F|$ нечетен, либо $G / F \simeq S_{3}$. Поэтому $l_{2}(G) \leqslant 2$. Следствие доказано.

СлЕДСТВИЕ 3. Пусть $G$ - группа, у которой индекс каждой максимальной подгруппы - либо простое число, либо квадрат простого числа. Тогда

(1) сверхразрешимый корадикал группы $G$ является расширением нильпотентной группы нечетного порядка с помощью 2 -группы и $n(G) \leqslant 4$;

(2) $l_{2}(G) \leqslant 2 u l_{p}(G) \leqslant 1$ для любого простого $p>2$.

ДокАЗАТЕЛьСтво. (1) По теоремеХолла [1, теорема VI.9.4] группа разрешима. Для рассматриваемой группы сохраняется доказательство теоремы 3 , только надо исключить случай, когда $|F|=p^{3}$. Поэтому сверхразрешимый корадикал групшы $G$ является расширением нильпотентной группы нечетного порядка с помощью 2 -группы и $n(G) \leqslant 4$;

(2) Из (1) следует, что группа $G$ обладает нормальным рядом $1 \triangleleft H \triangleleft G$ с метанильпотентными факторами $H$ и $G / H$. По лемме 5 получаем, что $l_{p}(G) \leqslant 2$ для любого простого $p$.

Для доказательства оценки $l_{p}(G) \leqslant 1$ при $p>2$ воспользуемся индукцией по порядку групшы $G$. Можно считать, что $\Phi(G)=O_{p^{\prime}}(G)=1$, а подгруппа Фиттинга $F=F(G)$ группы $G$ - единственная минимальная нормальная подгруппа группы $G$, которая является элементарной абелевой $p$-группой, и существует дополнение $M$ к подгрупе $F$ в группе $G$. Поэтому силовская $p$-подгруппа $G_{p}=[F]\left(G_{p} \cap M\right)=[F] M_{p}$, где $M_{p}-$ силовская $p$-подгруппа в $M$. Если $M_{p}=1$, то $F=G_{p}$ и $l_{p}(G) \leqslant 1$. Пусть $M_{p} \neq 1$. Так как $|F|=|G: M|$, то порядок $F$ равен $p$ либо $p^{2}$. 
Если $|F|=p$, то факторгруппа $G / F$ изоморфна подгруппе циклической групшы автоморфизмов Aut $F$ группы $F$, порядок которой равен $p-1$. Отсюда $G_{p}=F$ и $l_{p}(G) \leqslant 1$.

Пусть $|F|=p^{2}$. Тогда факторгруппа $G / F$ изоморфна подгруппе полной линейной группы $G L(2, p)$, порядок которой равен $\left(p^{2}-1\right)\left(p^{2}-p\right)$. Поэтому порядок силовской $p$-подгрупшы $G_{p}$ групшы $G$ равен $p^{3}$. Если $G_{p}$ абелева, то $l_{p}(G) \leqslant 1,[1$, c. 753$]$. Если $G_{p}$ неабелева, то она изоморфна либо метациклической группе, либо групше экспоненты $p[1$, с. 93$]$. Согласно лемме 4 первый случай невозможен, а по теореме ХоллаХигмэна $[11]$ во втором случае $l_{p}(G) \leqslant 1$.

Следствие 3 обобщает результаты Каморникова [9].

\section{СПИСОК ЦИТИРОВАННОЙ ЛИТЕРАТУРЫ}

[1] Huppert B. Endliche Gruppen I. Berlin-Heidelberg-New York: Springer, 1967.

[2] Мазуров В. Д. О конечных группах с метациклическими силовскими 2-подгруппами // Сиб. матем. ж. 1967. Т. 8. № 5. С. 966-982.

[3] Chillag D., Sonn J. Sylow-metacyclic groups and Q-admissibility // Israel J. Math. 1981. V. 40. № 3-4. P. 307-323.

[4] Huppert B. Über das Produkt von paarweise vertauschbaren zyklischen Gruppen // Math. Z. 1953. V. 58. P. 243-264.

[5] Maier R. Über die 2-nilpotentz faktorisierbarer endlicher Gruppen // Arch. Math. 1976. V. 27. № 5. P. 480-483.

[6] Gorenstein D. Finite Groups. New York, 1968.

[7] Шеметков Л. А. Формации конечных групп. М.: Наука, 1978.

[8] Супруненко Д. А. Группы матриц. М.: Наука, 1972.

[9] Каморников С. Ф. К теореме Ф. Холла // Вопросы алгебры. № 5. Минск: Университетское, 1990. С. 45-52.

[10] Guralnick R. Subgroups of prime power index in a simple group // J. Algebra. 1983. V. 81. № 2. P. 304-311.

[11] Hall P., Higman G. On the $p$-length of a $p$-soluble groups and reduction theorems for Burnside's problem // Proc. London Math. Soc. 1956. V. 3. P. 1-42.

Гомельский государственный университет им. $\Phi$. Скорины

Поступило

E-mail: monakhov@gsu.unibel.by

03.04 .2000

Исправленный вариант

05.12 .2000 